\title{
LOCAL DIOPHANTINE NULLSTELLEN INEQUALITIES
}

\author{
W. DALE BROWNAWELL
}

\section{INTRODUCTION}

Standard facts about resultants yield the following useful result.

If $P, Q \in \mathbf{Z}[x]$ are relatively prime polynomials of degree at most $D$ whose coefficients have height (maximum absolute value) at most $H$, then for any $\omega \in \mathbf{C}$,

$$
\max \{|P(\omega)|,|Q(\omega)|\} \geq(2 D H)^{-2 D} .
$$

A. O. Gelfond [Ge] built his method for algebraic independence on this simple inequality, which has other applications as well. The first extension to polynomials in several variables over $\mathbf{Z}$ was obtained by $D$. W. Masser and $G$. Wüstholz as a corollary to their effective version of the Hilbert Nullstellensatz [Ma-Wü, Theorem IV]:

Theorem (Masser-Wüstholz). Let $P_{1}, \ldots, P_{k} \in \mathbf{Z}\left[x_{1}, \ldots, x_{m}\right]$ of degree $\leq D$ and $\log$ height $\leq h$ have no common zeros in $\mathbf{C}^{m}$. Then there are $a \in \mathbf{N}$ and $A_{1}, \ldots, A_{k} \in \mathbf{Z}\left[x_{1}, \ldots, x_{m}\right]$ of degree $\leq(8 D)^{2 M+1}$, where $M=2^{m-1}$, such that

$$
\begin{gathered}
a=A_{1} P_{1}+\cdots+A_{k} P_{k}, \\
\log a, \operatorname{loght} A_{i} \leq(8 D)^{4 M-1}(h+8 D \log 8 D) .
\end{gathered}
$$

The obvious upper bound for $\left|A_{i}(\omega)\right|$ then gives [Ma-Wü, Lemma 7, p. 459]

$$
\log \max \left\{\left|P_{i}(\omega)\right|\right\} \geq-(8 D)^{4 M+1}(h+8 D \log 8 D)-(8 D)^{2 M+1} \log |\omega|,
$$

where $|\omega|:=\max \left\{1,\left|\omega_{i}\right|\right\}$ for any $\omega=\left(\omega_{1}, \ldots, \omega_{m}\right) \in \mathbf{C}^{m}$. The bound on the height in the theorem follows rather directly from the bound on the degree. It was conjectured that a solution of (1.2) should always exist satisfying much smaller bounds, which would significantly improve (1.3). Very recently, in work [Br2] done after this manuscript was essentially completed, the author has found an explicit solution of (1.2) over $\mathbf{C}$ with degrees at most $3 \mathrm{~m}^{2} \mathrm{D}^{m}$ (and, say, $a=$ 1). This bound is optimal up to the constant factor $3 \mathrm{~m}^{2}$, as follows [Br3] from the example below of Masser and P. Philippon. However, since the coefficients

Received by the editors December 23, 1986.

1980 Mathematics Subject Classification (1985 Revision). Primary $11 \mathrm{~J} 25$.

Research supported in part by the NSF. 
of the $A_{i}$ are given as integrals in an identity pointed out by C. Berenstein and A. Yger, one loses control of their arithmetic properties. Moreover when working over $\mathbf{Z}$, the procedure of Masser-Wüstholz applies Cramer's rule to the approximately $D^{m^{2}} \times D^{m^{2}}$ system of inhomogeneous equations over $\mathbf{Z}$ furnished by our bounds on $\operatorname{deg} A_{i}$. Solving gives coefficients of $A_{i}$ with height at most $\exp \left(c D^{m^{2}}(h+\log D)\right)$, and thus

$$
\log \max \left\{\left|P_{i}(\omega)\right|\right\} \geq-c D^{m^{2}}(h+\log D+\log |\omega|),
$$

for some $c>0$, depending on $m$.

The purpose of the present note is to establish the following even sharper local version of inequality (1.3).

Theorem A. Let $P_{1}, \ldots, P_{n} \in \mathbf{Z}\left[x_{1}, \ldots, x_{m}\right]$ have total degree at most $D$ and $\log$ height at most $h$. If $P_{1}, \ldots, P_{n}$ have no common zeros within $\rho, 0<\rho \leq 1$, of $\omega \in \mathbf{C}^{m}$, then

$$
\log \max \left\{\left|P_{i}(\omega)\right|\right\} \geq-D^{\mu}\left\{c_{1} D+c_{2} h\right\}-c_{2} D^{\nu} \log \left(|\omega|^{2} / \rho\right),
$$

where $\mu=\min \{m, n-1\}, \nu=\min \{m, n\}, c_{1}=11(m+1)^{5}, c_{2}=(m+1)^{2}$.

Corollary A. In the situation of Theorem A,

$$
\log \max \left\{\left|P_{i}(\omega)\right|\right\} \geq-D^{m}\left\{c_{1} D+c_{2}\left(h+\log \left(\rho /|\omega|^{2}\right)\right)\right\} .
$$

This sort of result seems to have been unnoticed even for $m=1$, although that case can be deduced from properties of the semiresultant of G. V. Chudnovsky [Ch, Brl]. The result of Theorem $\mathrm{A}$ can be sharpened when any ideal $\mathfrak{A}$ of polynomials over $\mathbf{Z}$ is known to be small at $\omega$ in an appropriate sense. Theorem B of $\S I I$ gives this generalization.

In addition to their appeal as new types of inequalities in diophantine approximations, these results provide an alternative to the Gelfond-Philippon approach [ $\mathrm{Ph} 1, \mathrm{Ph} 2]$ to algebraic independence involving an infinite sequence of ideals. See $[\mathrm{Br} 3, \mathrm{Br}-\mathrm{Tu}]$ for a more complete discussion, which shows how to weaken, albeit not eliminate, the usual technical hypothesis involving measures of linear independence.

The reader will notice that with little difficulty the constants $c_{i}$ in the statement of our theorems can be improved somewhat. The main results of this paper hold for nonarchimedean valuations as well, with only a change in the constants. One has only to adopt the definitions and invoke the lemmas of [Ph1] corresponding to those used here for their relative ease of statement.

I am grateful to D. W. Masser for suggesting during the early stages of research on the initial, global result that local inequalities might be useful. I am also indebted to W. Schmidt and M. Waldschmidt for the remark that the example $P_{1}=x_{1}{ }^{D}, \quad P_{2}=x_{1}-x_{2}{ }^{D}, \ldots, P_{m-1}=x_{m-2}-x_{m-1}{ }^{D}, \quad P_{m}=1-x_{m-1} x_{m}{ }^{D-1}$ 
of Masser and Philippon evaluated at the point $\omega=\left(t^{-D^{m-1}+D^{m-2}}, \ldots, t^{-D+1}, t\right)$ with $t>1$ satisfies $\max \left\{\left|P_{i}(\omega)\right|\right\}=|\omega|^{-D^{m}+D^{m-1}}$. Thus the coefficient of $-D^{\nu} \log |\omega|$ must be at least 1 in Theorem A. Variations of this example

(i) where $P_{m}=x_{m-1}-\left(q x_{m}-p\right)^{D}$ for a sequence $\{p / q\}$ of good rational approximations to a fixed Liouville number or

(ii) where $P_{m}=x_{m}{ }^{D}-x_{m-1}, P_{m+1}=H x_{m}-1$ for $H$ a large positive integer

show that nowhere else in the statement of the theorem can $c_{2}$ be taken $<1$.

\section{BASIC RESULTS}

To state Theorem $\mathrm{B}$ and the basic underlying proposition, we need some terminology for homogeneous ideals in $\mathbf{Z}[\underline{x}]=\mathbf{Z}\left[x_{0}, \ldots, x_{m}\right]$. For $1 \leq j \leq m$, we introduce the linear forms

$$
L_{j}(\underline{x})=u_{j 0} x_{0}+\cdots+u_{j m} x_{m},
$$

in the new variables $u_{j k}, 0 \leq k \leq m$. For any homogeneous ideal $\mathfrak{A}<\mathbf{Z}[\underline{x}]$, and any positive integer $d$, consider the ideal $\mathfrak{A}^{\prime}(d)<\mathbf{Z}\left[\underline{x}, \underline{u}_{1}, \ldots, \underline{u}_{d}\right]$, where $\underline{u}_{j}:=\left(u_{j 0}, \ldots, u_{j m}\right)$ for $j=1, \ldots, d \leq m$, consisting of those polynomials $G$ for which there is a positive integer $M \in \mathbf{N}$ such that for all $\mu=0, \ldots, m$,

$$
G x_{\mu}{ }^{M} \in\left(\mathfrak{A}, L_{1}, \ldots, L_{d}\right) \text {. }
$$

We will be most concerned with the properties of

$$
\mathfrak{A}^{\prime \prime}(d):=\mathfrak{A}^{\prime}(d) \cap \mathbf{Z}_{d},
$$

where $\mathbf{Z}_{d}:=\mathbf{Z}\left[\underline{u}_{1}, \ldots, \underline{u}_{d}\right]$, which are developed in [Ne1, Ne2, Ne3, Ph1, Ph3].

Lemma 1 ([Ne1, Lemma 5]). Let $\mathfrak{A}$ be an unmixed homogeneous ideal of $\mathbf{Z}[\underline{x}]$ of rank $r$.

(i) If $r \leq m-d$, then $\mathfrak{A}^{\prime \prime}(d)=(0)$.

(ii) If $r=m-d+1$, then $\mathfrak{A}^{\prime \prime}(d)$ is a nonzero principal ideal of $\mathbf{Z}_{d}$.

When $d=m+1-\operatorname{rank}(\mathfrak{A})$, we call a generator $F$ of $\mathfrak{A}^{\prime \prime}(d)$ an (integral) Chow form of $\mathfrak{A}$. Following Nesterenko [Ne2], we then define the degree and height of $\mathfrak{A}$ in terms of $F$ :

$$
\delta_{\mathfrak{A}}:=\delta_{F}:=\operatorname{deg}_{1} F, \quad \chi_{\mathfrak{A}}:=\chi_{F}:=\log H_{F}:=\log \text { height } F,
$$

where $\operatorname{deg}_{1} F$ is the total degree of $F$ with respect to $\underline{u}_{1}=\left(u_{10}, \ldots, u_{1 m}\right)$. Note that this definition of $\delta_{\mathfrak{a}}$ agrees with the usual definition of the degree of an ideal only when the length of each primary component equals its exponent (see Lemma 2 in $\S$ III). For $\omega=\left(\omega_{0}, \ldots, \omega_{m}\right) \in \mathbf{C}^{m+1}$ with $\|\omega\|:=\max \left|\omega_{i}\right|>$ 0 , define the absolute value of the Chow form $F$ at $\omega$ in the following way. For $j=1, \ldots, d$ with $d=m+1-\operatorname{rank}(\mathfrak{A})$, let $S^{(j)}=\left(s_{k l}^{(j)}\right)$ be skew symmetric matrices in the new variables $s_{k l}^{(j)}, 0 \leq k<l \leq m$. Then we set

$$
\|\mathfrak{A}\|_{\omega}:=\|F\|_{\omega}:=H\left(F\left(S^{(1)} \omega, \ldots, S^{(d)} \omega\right)\right)\|\omega\|^{-d \delta_{F}},
$$


where $H$ denotes the height of $F\left(S^{(1)} \omega, \ldots, S^{(d)} \omega\right)$ considered as a polynomial over $\mathbf{C}$ in the variables $s_{k l}^{(j)}, 0 \leq k<l \leq m, j=1, \ldots, d$. This definition can perhaps be rendered more palatable by noting that at least when $\mathfrak{A}$ is a prime ideal, then the coefficients appearing in $F\left(S^{(1)} \underline{x}, \ldots, S^{(d)} \underline{x}\right)$ are homogeneous polynomials from $\mathbf{Z}[\underline{x}]$ which generate $\mathfrak{A}$ or its intersection with some embedded components or some isolated components meeting $\mathbf{Z}$ (see [Ne1, Lemma 11]).

To state our basic result we use the notation that

$$
\|Q\|_{\omega}:=|Q(\omega)| \cdot\|\omega\|^{-\operatorname{deg} Q}
$$

for homogeneous $Q \in \mathbf{Z}[\underline{x}]$ and, for $\rho>0$ and nonzero $\omega \in \mathbf{C}^{m+1}$,

$$
B_{\rho}(\omega):=\left\{\theta \in \mathbf{C}^{m+1} \backslash\{0\}: \max \left|\theta_{i} \omega_{j}-\theta_{j} \omega_{i}\right|<\rho\|\omega\|\|\theta\|\right\},
$$

where $\theta=\left(\theta_{0}, \ldots, \theta_{m}\right)$ and $\|\theta\|=\max \left|\theta_{i}\right| . B_{\rho}(\omega)$ is a sort of open projective ball of radius $\rho$.

Theorem B. Let $\mathfrak{A}$ be an unmixed homogeneous ideal of $\mathbf{Z}\left[x_{0}, \ldots, x_{m}\right]$ and $R_{1}, \ldots, R_{n}$ be homogeneous polynomials, $\operatorname{deg} R_{i} \leq D$ and $\log \mathrm{ht} R_{i} \leq h$, with no zeros in common with $\mathfrak{A}$ inside $B_{\rho}(\omega)$ for nonzero $\omega \in \mathbf{C}^{m+1}, \rho \leq 1$. Then

$$
\log \max \left\{\|\mathfrak{A}\|_{\omega},\left\|R_{i}\right\|_{\omega}\right\} \geq-D^{\mu}\left\{c_{1}{ }^{\prime} D+c_{2}{ }^{\prime} h\right\}+D^{\nu} c_{2} \log \rho
$$

where $\mu=\min \{n-1, \operatorname{dim} \mathfrak{A}), \nu=\min \{n, \operatorname{dim} \mathfrak{A}\}, c_{1}{ }^{\prime}=10 m^{2}(m+1)^{3} \operatorname{deg} \mathfrak{A}+$ $c_{2} \log \mathrm{ht} \mathfrak{A}$, and $c_{2}^{\prime}=c_{2} \operatorname{deg} \mathfrak{A}$.

Corollary B. If in the situation of Theorem $\mathrm{A}, \mathfrak{B}$ is an unmixed ideal of ordinary integral polynomials vanishing at $\omega \in \mathbf{C}^{m}$, then

$$
\log \max \left\{\left|P_{i}(\omega)\right|\right\} \geq-D^{\mu}\left\{c_{1}{ }^{\prime} D+c_{2}{ }^{\prime} h\right\}-c_{2} D^{\nu} \log \left(|\omega|^{2} / \rho\right),
$$

where $\mu=\min \{n-1, \operatorname{dim} \mathfrak{B}\}, \nu=\min \{n, \operatorname{dim} \mathfrak{B}\}, c_{1}{ }^{\prime}=c_{1} \operatorname{deg}^{h} \mathfrak{B}+$ $c_{2} \log \mathrm{ht}^{h} \mathfrak{B}, c_{2}{ }^{\prime}=c_{2} \operatorname{deg}^{h} \mathfrak{B}$ and ${ }^{h} \mathfrak{B}$ is the homogenization of $\mathfrak{B}$.

The key to the proof of these results concerns lower bounds on polynomials which form a sort of regular sequence if we restrict our attention to a rather small neighborhood of $\omega$.

Proposition. Let $\mathfrak{A}$ be an unmixed homogeneous ideal of $\mathbf{Z}\left[x_{0}, \ldots, x_{m}\right]$ of rank $m+1-d$, degree $D_{d}, D_{d}>0$, and $\chi_{\mathfrak{A}} \leq h_{d}$. Let $\omega \in \mathbf{C}^{m+1}$ be nonzero and $0<\rho \leq 1$. For $j=d-1, d-2, \ldots, d^{\prime}$, let $Q_{j}$ be a homogeneous polynomial with $\operatorname{deg} Q_{j} \leq D_{j}$ and $\log \mathrm{ht} Q_{j} \leq h_{j}$. Assume for each $j$ that $Q_{j}$ does not lie in any isolated prime ideal of $\mathfrak{A}_{j+1}:=\left(\mathfrak{A}, Q_{d-1}, \ldots, Q_{j+1}\right)$ of dimension $j$ which has zeros inside $B_{\rho}(\omega)$. If no isolated prime ideal of $\mathfrak{A}_{d^{\prime}}:=\left(\mathfrak{A}, Q_{d-1}, \ldots, Q_{d^{\prime}}\right\}$ of dimension $d^{\prime}-1$ has zeros in $B_{\rho}(\omega)$, then the 
following inequality holds:

$$
\begin{aligned}
& \log \max \left\{\|\mathfrak{A}\|_{\omega},\left\|Q_{d-1}\right\|_{\omega}, \ldots,\left\|Q_{d^{\prime}}\right\|_{\omega}\right\} \\
& \quad \geq-D_{d} \cdots D_{d^{\prime}}\left(10 m^{3} d^{2}+\sum_{i=d^{\prime}}^{d} i h_{i} / D_{i}\right)+d^{2} D_{d} \cdots D_{d^{\prime \prime}} \log \rho,
\end{aligned}
$$

where $d^{\prime \prime}:=\max \left\{1, d^{\prime}\right\}$.

Remark 1. When $d^{\prime}=0$, then the condition on $\mathfrak{A}_{0}$ is vacuously satisfied. If $\mathfrak{A}=(0)$, then we take $D_{d}=1, h_{d}=0$.

Remark 2. We can actually prove the Proposition in a somewhat more precise form by interpreting, as in [Ph2], the sense of a prime ideal $\mathfrak{R}$ of dimension $d-1$ "having zeros in $B_{\rho}(\omega$ " as meaning that

$$
\text { “ }\|\Re\|_{\omega} \leq \rho^{d \delta_{\mathfrak{R}}} e^{-3 m^{3} d \delta_{\mathfrak{s}}} . "
$$

Remark 3. The proof of this lower bound extends naturally to fields with finite transcendence type. We plan to undertake this generalization in a separate paper, since the necessary lemmas are not yet in the literature.

However, we note that the theorems generalize almost effortlessly to allow coefficients from an algebraic number field $K=\mathbf{Q}(\zeta)$, say, where $\zeta$ has monic minimal polynomial $f$ over $\mathbf{Z}$ of degree $\delta$ and height $H_{\zeta}$. For $\alpha=\sum_{i=0}^{\delta-1} a_{i} \zeta^{i}$ $\in \mathbf{Z}[\zeta]$, define the (naive) height of $\alpha$ with respect to $\zeta$ to be $\max \left|a_{i}\right|$. For a polynomial $P$ over $\mathrm{Z}[\zeta]$, we say that the naive height of $P$ is the maximal naive height of its coefficients. Finally we say that size $P$ is the sum of its total degree and the logarithm of its naive height. For simplicity we give the extension of Theorem A only.

Theorem $\mathrm{A}^{\prime}$. Let $P_{1}, \ldots, P_{n} \in \mathrm{Z}[\zeta]\left[x_{1}, \ldots, x_{m}\right]$ have total degree at most $D$ and size at most $T$. Let $P_{1}, \ldots, P_{n}$ have no common zeros within $B_{\rho}(\omega)$, where $0<\rho<\left(\delta H_{\zeta}\right)^{-2 \delta}, \omega=\left(\omega_{1}, \ldots, \omega_{m}\right) \in \mathbf{C}^{m}$. Then

$$
\left.\log \max \left\{\left|P_{i}(\omega)\right|\right\} \geq-c_{1}{ }^{\prime \prime} D^{\mu} T-c_{2}{ }^{\prime \prime} D^{\nu} \log \left(\left|\omega^{\prime}\right|^{2} / \rho\right)\right\},
$$

where $\left|\omega^{\prime}\right|:=\max \left\{1,|\zeta|,\left|\omega_{i}\right|\right\}$ and $c_{1}{ }^{\prime \prime}, c_{2}{ }^{\prime \prime}$ are effective constants depending only on $H_{\zeta}, \delta$, and $m$.

\section{AUXILIARY PROPOSITIONS}

Before we give the rather short proof of our Basic Proposition, we recall the fundamental properties of integral Chow forms. All ideals in this section will be homogeneous ideals in $\mathbf{Z}\left[x_{0}, \ldots, x_{m}\right]$ unless otherwise specified. The next two lemmas describe how a Chow form of an unmixed ideal $\mathfrak{A}$ is related to the zeros of $\mathfrak{A}$ lying on a "generic" linear subspace of dimension $d-1=m-\operatorname{rank}(\mathfrak{A})$. 
Lemma 2 ([Ne1, Corollary of Proposition $\left.\left.3^{\prime}\right]\right)$. Let $\mathfrak{A}$ be unmixed with primary decomposition $\mathfrak{A}=\mathfrak{Q}_{1} \cap \cdots \cap \mathfrak{Q}_{t}$, where $\mathfrak{Q}_{i}$ is $\mathfrak{R}_{i}$-primary of exponent $e_{i}$ and $\mathfrak{R}_{i} \cap \mathbf{Z}=(0), i=1, \ldots, s$, whereas $\mathfrak{Q}_{s+1} \cap \cdots \cap \mathfrak{Q}_{t} \cap \mathbf{Z}=(b), b \in \mathbf{N}$. Let $F_{i}$ be a Chow form for $\mathfrak{R}_{i}, i=1, \ldots, s$. Then

$$
F=b F_{1}^{e_{1}} \cdots F_{s}^{e_{s}}
$$

is a Chow form for $\mathfrak{A}$.

Thus to understand the general unmixed situation we need to understand only the prime case. For the next lemma, we set $k_{d-1}:=\mathbf{Q}\left(\underline{u}_{1}, \ldots, \underline{u}_{d-1}\right)$.

Lemma 3 ([Ne2, Lemma 2]). Let $\mathfrak{R}$ be a prime ideal of rank $m+1-d, x_{0} \notin \Re$, $\mathfrak{R} \cap \mathbf{Z}=(0)$, with Chow form $F$. Then there is a finite Galois extension $L$ of $k_{d-1}$ such that in $L\left[\underline{u}_{1}, \ldots, \underline{u}_{d}\right]$, we can factor $F$ as

$$
F=a \prod_{\gamma}\left(u_{d 0}+\alpha_{1}^{\gamma} u_{d 1}+\cdots+\alpha_{m}{ }^{\gamma} u_{d m}\right),
$$

where $\gamma$ runs over all the $k_{d-1}$-embeddings of $k_{d-1}\left(\alpha_{1}, \ldots, \alpha_{m}\right)$ into an algebraic closure and where $a \in \mathbf{Z}\left[\underline{u}_{1}, \ldots, \underline{u}_{d-1}\right]$.

Lemma 5 below gives the basic resultant construction of Nesterenko on which this paper rests. However, in contrast to Nesterenko and Philippon, our philosophy is to take a Chow form rather than an ideal as our point of departure in induction. Whether we start with an underlying unmixed ideal in mind or not, we call any product $F$ of Chow forms of prime ideals of the same rank $m-d+1$ a Chow form of that rank, or of dimension $d-1$. Of course, given a Chow form in this general sense, one can always concoct an ideal for which it is a Chow form in the original sense by means of symbolic powers. We define $\delta_{F}:=\operatorname{deg}_{\underline{u}_{1}} F, \chi_{F}:=\log$ height $F$ and for nonzero $\omega \in \mathbf{C}^{m+1}$,

$$
\|F\|_{\omega}:=\left(\text { height } F\left(S^{(1)} \omega, \ldots, S^{(d)} \omega\right)\right)\|\omega\|^{-d \delta_{F}} .
$$

Unfortunately height $F$ and $\|F\|_{\omega}$ are not multiplicative functions of $F$, unlike Philippon's corresponding definitions [Ph1] in terms of Mahler measures. This is only a minor nuisance because of Gelfond's inequality.

Lemma 4 ([Ge, Lemma II, p. 135]). Let $G_{1}, \ldots, G_{s} \in \mathbf{C}\left[y_{1}, \ldots, y_{t}\right]$ have heights $H_{1}, \ldots, H_{s}$ and $G=G_{1} \cdots G_{s}$ have height $H$. Then

$$
H_{1} \cdots H_{s} \leq H e^{\Delta}
$$

where $\Delta:=\sum_{i=1}^{t} \operatorname{deg}_{y_{i}} G$.

We now turn to the basic properties of the resultant of a Chow form and a polynomial outside every associated prime of the Chow form. For the next lemmas, we fix notation in the following way: $\mathfrak{R}_{1}, \ldots, \mathfrak{R}_{s}$ are prime ideals of 
$\mathbf{Z}[\underline{x}]$ of rank $m-d+1$ with $\mathfrak{R}_{i} \cap \mathbf{Z}=(0), i=1, \ldots, s$. The corresponding Chow forms are

$$
F_{i}=a_{i} \prod_{\gamma \in \Gamma_{i}}\left(\alpha_{i 0}{ }^{\gamma} u_{d 0}+\cdots+\alpha_{i m}{ }^{\gamma} u_{d m}\right),
$$

where for each $i$ there is a $k$ such that $\alpha_{i k}{ }^{\gamma}=1$ as $\gamma$ runs through $\Gamma_{i}$, the set of all embeddings of some $k_{d-1}\left(\alpha_{i 0}{ }^{\gamma_{0}}, \ldots, \alpha_{i m}{ }^{\gamma_{0}}\right)$ into a fixed algebraic closure of $k_{d-1}$. Let $q_{1}, \ldots, q_{s} \in \mathbf{N}$ and set

$$
F=F_{1}^{q_{1}} \cdots F_{s}^{q_{s}} .
$$

We shall sometimes refer to the quantity $G$ introduced in (3.2) as the resultant of the Chow form $F$ and the ordinary form $Q$. It was devised by Nesterenko in $[\mathrm{Ne} 2]$.

Lemma 5. Let $Q \in \mathbb{Z}\left[x_{0}, \ldots, x_{m}\right]$ be homogeneous and lie outside all $\mathfrak{R}_{i}$, $i=1, \ldots, s$. Set

$$
G=\prod_{i=1}^{s}\left(a_{i}^{\operatorname{deg} Q} \prod_{\gamma \in \Gamma_{i}} Q\left(\alpha_{i 0}{ }^{\gamma}, \ldots, \alpha_{i m}{ }^{\gamma}\right)\right)^{q_{i}} .
$$

Then $G \in \mathbf{Z}\left[\underline{u}_{1}, \ldots, \underline{u}_{d-1}\right]$. Let $\mathfrak{p}_{1}, \ldots, \mathfrak{p}_{t}$ be a complete list of all minimal prime ideals such that $\mathfrak{p}_{j}$ is associated to some $\left(\mathfrak{R}_{i}, Q\right)$ and $\mathfrak{p}_{j} \cap \mathbf{Z}=(0)$. Then there are $b, f_{1}, \ldots, f_{t} \in \mathbf{N}$ such that

$$
G= \pm b E_{1}{ }^{f_{1}} \cdots E_{t}^{f_{t}},
$$

where $E_{j}$ is a Chow form of $\mathfrak{p}_{j}$, i.e. $G$ is a Chow form of rank $m+2-d$. Moreover let $\delta_{Q}:=\operatorname{deg}_{\underline{x}} Q, \chi_{Q}:=\log H_{Q}$, where $H_{Q}=$ height $Q$. Then

(i) $\delta_{G}=\delta_{F} \delta_{Q}$.

(ii) $\chi_{G} \leq \delta_{Q} \chi_{F}+\delta_{F} \chi_{Q}+(m+2 d \log (m+1)) \delta_{F} \delta_{Q}$.

Here we mean that if $d=1$, then $G \in \mathbf{Z}$ and property (i) holds vacuously. For the case $s=q_{1}=1$, the assertions of Lemma 5 were proved by Nesterenko in Lemmas 5 and $6[\mathrm{Ne} 3]$ with an inequality in (i) and $d$ in inequality (ii) instead of $2 d$. Equality in (i) is established in [ $\mathrm{Br} 2]$ (and implicitly already in [Ph3]). The claims then follow on applying those results to each $G_{i}$,

$$
G_{i}=a_{i}^{\delta_{Q}} \prod_{\gamma \in \Gamma_{i}} Q\left(\alpha_{i 0}{ }^{\gamma}, \ldots, \alpha_{i m}{ }^{\gamma}\right),
$$

in $G=\prod_{i=1}^{s} G_{i}^{q_{i}}$ and noting that each $G_{i}$ involves at most $(m+1)^{(d-1) \delta_{F} \delta_{Q}}$ monomials.

Lemma 6. Under the conditions of Lemma 5, if $\omega \in \mathbf{C}^{m+1}$ is nonzero, then

$$
\log \|G\|_{\omega} \leq \delta_{F} \chi_{Q}+\delta_{Q} \chi_{F}+6 m^{2} \delta_{F} \delta_{Q}+\log \max \left\{\|F\|_{\omega} / H_{F},\|Q\|_{\omega} / H_{Q}\right\},
$$

where $H_{F}=$ height $F$. 
Proof. The proof is obtained by repeating the argument for Proposition 3 of [Ne3], this time for general $s$ and $q_{i}$. A somewhat weaker statement can be deduced directly from (28) of the proof of that proposition, which implies that

$$
\log \|G\|_{\omega} \leq \delta_{F} \chi_{Q}+\delta_{Q} \chi_{F}+6 m^{2} \delta_{F} \delta_{Q}+\sum_{i=1}^{t} q_{i} \log \max \left\{\left\|F_{i}\right\|_{\omega} / H_{F_{i}},\|Q\|_{\omega} / H_{Q}\right\},
$$

if in the two steps immediately preceding (28), the extra terms corresponding to $H_{F_{i}}, H_{Q}$ are not ignored (see also [Ne4]). But by Gelfond's inequality (3.1), since clearly

$$
\max \left\{\left\|F_{i}\right\|_{\omega} / H_{F},\|Q\|_{\omega} / H_{Q}\right\} \leq \max \left\{e^{m\left(\operatorname{deg} F_{i}\right)},(1+m)^{\delta_{Q}}\right\},
$$

we see that we obtain the desired inequality with an extra term of $2 m d \delta_{F} \delta_{Q}$ on the right-hand side. We content ourselves with the indication of this somewhat weaker result.

The next result was first proved by Philippon to treat the "second case" in his elimination procedure [ $\mathrm{Ph} 1]$. Nevertheless we cite it in a form adapted to Nesterenko's definitions given in Lemma 6 of [ $\mathrm{Ne} 4]$.

Lemma 7. Let $\mathfrak{A}$ be an unmixed ideal of $\mathbf{Z}\left[x_{0}, \ldots, x_{m}\right]$ of rank $m-d+1 \leq m$, $\mathfrak{A} \cap \mathbf{Z}=(0)$. If $\mathfrak{A}$ has no zero in $B_{\rho}(\omega)$, for nonzero $\omega \in \mathbf{C}^{m+1}$ and $0<\rho \leq 1$, then

$$
\rho^{d \delta_{\mathrm{x}}} \leq\|\mathfrak{A}\|_{\omega} e^{3 m^{3} d \delta_{\mathrm{X}}} .
$$

Lemma 8. If under the conditions of Lemma 5 we denote by $G^{*}$ the Chow form obtained from $G$ by omitting from $G$ all factors from $\mathbf{Z}$ and those factors coming from prime ideals having no zeros inside $B_{\rho}(\omega)$, then either $G^{*}= \pm 1$ or else

(i) $\delta_{G^{*}} \leq \delta_{F} \delta_{Q}$,

(ii) $\chi_{G^{*}} \leq \delta_{F} \chi_{Q}+\delta_{Q} \chi_{F}+m(3 d+1) \delta_{F} \delta_{Q}$.

In any case,

(iii) $\log \left\|G^{*}\right\|_{\omega} \leq \delta_{F} \chi_{Q}+\delta_{Q} \chi_{F}+6 m^{3} d \delta_{F} \delta_{Q}+\log \max \left\{\|F\|_{\omega} / H_{F},\|Q\|_{\omega} / H_{Q}\right\}-$ $(d-1) \delta_{F} \delta_{Q} \log \rho$.

Proof. The first inequality is obvious from Lemma 5. The second follows from part (ii) of Lemma 5 and Gelfond's inequality (3.1). The third inequality follows from Lemmas 6, 7, and Gelfond's inequality.

Lemma 9 (cf. [Ne3, Proposition 1]). Let $\mathfrak{A}=(P)$ with $P \in \mathbb{Z}\left[x_{0}, \ldots, x_{m}\right]$ nonzero and homogeneous. Then

(i) $\delta_{\mathfrak{A}}=\delta_{P}, \chi_{\mathfrak{A}} \leq \chi_{P}+m^{2} \delta_{P}$,

(ii) $\|\mathfrak{A}\|_{\omega} \leq\|P\|_{\omega}(m+1)^{2 m \delta_{P}}$. 


\section{PROOFS OF RESULTS}

A. Proof of Proposition. Our proof of the Proposition will be a sort of decreasing induction on the dimension. We shall consider an assertion $H_{k}$ for each $k$ in the range $\min \{d, m\}=: m^{\prime} \geq k \geq d^{\prime}$. We shall establish $H_{m^{\prime}}$, and for $k>d^{\prime}$ we show that $H_{k}$ implies either the inequality of our proposition or $H_{k-1}$. But $H_{d^{\prime}}$ also easily implies our inequality, and therefore the inequality will be established in all cases. For $m^{\prime} \geq k \geq d^{\prime}$ the statements are the following, where each sum runs over $i$ (and $j$ if it actually appears in the summand) in the range $k \leq i \leq d$ (or $k \leq i \leq j \leq d$ ), excepting $\sum^{\prime}$, which is over $\max \{k, 1\} \leq i \leq d$.

$H_{k}$ : There is a Chow form $F_{k}$, of dimension $k-1$ if $F_{k} \neq \pm 1$, whose underlying prime ideals are the isolated prime ideals of $\mathfrak{A}_{k}=\left(\mathfrak{A}, Q_{d-1}, \ldots, Q_{k}\right)$ with zeros inside $B_{\rho}(\omega)$, such that

(i) $\operatorname{deg} F_{k} \leq D_{d} \cdots D_{k}$,

(ii) $\log$ height $F_{k} \leq \sum h_{i} D_{d} \cdots \hat{D}_{i} \cdots D_{k}+m D_{d} \cdots D_{k} \sum(3 i+1)$,

(iii)

$$
\begin{aligned}
\log \left\|F_{k}\right\|_{\omega} \leq & \sum h_{j} D_{d} \cdots \hat{D}_{j} \cdots D_{i}+m \sum(3 j+1) D_{d} \cdots D_{i} \\
& +6 m^{3} \sum i D_{d} \cdots D_{i}-\sum(i-1) D_{d} \cdots D_{i} \log \rho \\
& +\log \max \left\{\|\mathfrak{A}\|_{\omega},\left\|Q_{d-1}\right\|_{\omega}, \ldots,\left\|Q_{k}\right\|_{\omega}\right\} .
\end{aligned}
$$

Whenever $F_{k}= \pm 1$, assertions (i) and (ii) hold automatically and part (iii) implies the inequality desired. Since by hypothesis $\mathfrak{A}_{d^{\prime}}$ has no zeros of dimension $d^{\prime}-1$ in $B_{\rho}(\omega), F_{d^{\prime}}= \pm 1$ if no previous $F_{k}= \pm 1$. We consider two cases to start the induction.

$d \leq m$ : Here $\mathfrak{A} \neq(0)$, and we let $F_{d+1}{ }^{*}$ be a Chow form of $\mathfrak{A}$. On deletion of the factors of $F_{d+1}{ }^{*}$ whose prime ideals have no zeros inside $B_{\rho}(\omega)$, we apply Gelfond's inequality (3.1) and Lemmas 2 and 7 to obtain a stronger version of $H_{d} \quad\left(=H_{m^{\prime}}\right)$.

$d-1=m$ : Here $\mathfrak{A}=(0)$, and we let $F_{m}{ }^{*}$ be a Chow form of $\left(Q_{m}\right)$, with $\operatorname{deg}{F_{m}}^{*}$, ht ${F_{m}}^{*},\left\|F_{m}{ }^{*}\right\|_{\omega}$ estimated by Lemma 9 . We delete all factors from $F_{m}{ }^{*}$ whose prime ideals have no zeros inside $B_{\rho}(\omega)$ to obtain $F_{m}$. We apply (3.1) and Lemmas 2 and 7 to show $H_{m}\left(=H_{m^{\prime}}\right)$ to be true.

For the induction step we consider $k$ in the interval $d^{\prime} \leq k<m^{\prime}$. We assume that $H_{k+1}$ has been established. If $F_{k+1} \in \mathbf{Z}$ (and thus $F_{k+1}= \pm 1$ ), the claim of the Proposition is established by (iii). If $F_{k+1} \notin \mathbf{Z}$, then it is also a Chow form of dimension $k$, and the prime ideals $\mathfrak{R}$ underlying $F_{k+1}$ are all the isolated prime ideals of $\mathfrak{A}_{k+1}=\left(\mathfrak{A}, Q_{d-1}, \ldots, Q_{k+1}\right)$ having zeros within $B_{\rho}(\omega)$. Since however $Q_{k}$ is not contained in any isolated prime ideal of $\mathfrak{A}_{k+1}$ of dimension $k$ having zeros inside $B_{\rho}(\omega)$, the resultant $F_{k}^{*}=\operatorname{Res}\left(F_{k+1}, Q_{k}\right) \not \equiv 0$, and we apply Lemma 8 to obtain $F_{k}$ verifying conditions (i), (ii), (iii). Notice that when $k=0$, we apply Lemma 6 instead. This accounts for $\sum^{\prime}$. 
The only remaining question concerns the prime ideals underlying $F_{k}$ when $k>0$. By construction, each prime ideal $\mathfrak{R}$ underlying $F_{k}$ contains $Q_{k}$ and some prime $\mathfrak{p}$ of $F_{k+1}$, which by induction is a prime of $\mathfrak{A}_{k+1}$. Now every prime $\mathfrak{p}$ of $\mathfrak{A}_{k+1}$ contained in $\mathfrak{R}$ has zeros in $B_{\rho}(\omega)$, since $\mathfrak{R}$ itself does, and thus by induction underlies $F_{k+1}$. Since $Q_{k}$ is contained in no such $\mathfrak{p}$ by construction and $\operatorname{dim} \mathfrak{R}+1=\operatorname{dim} \mathfrak{p}, \mathfrak{R}$ is an isolated prime of every such $\left(\mathfrak{p}, Q_{k}\right.$ ) and thus of $\mathfrak{A}_{k}=\left(\mathfrak{A}_{k+1}, Q_{k}\right)$ (and by construction has zeros in $B_{\rho}(\omega)$ ).

Conversely let $\mathfrak{A}$ be an isolated prime ideal of $\mathfrak{A}_{k}$ having zeros in $B_{\rho}(\omega)$. Then since $\mathfrak{A}_{k}=\left(\mathfrak{A}_{k+1}, Q_{k}\right), \mathfrak{R}$ is in particular a minimal prime of $\left(\mathfrak{p}, Q_{k}\right)$ for some prime $\mathfrak{p}$ of $\mathfrak{A}_{k+1}$. In addition $\mathfrak{p}$ has zeros in $B_{\rho}(\omega)$ since $\mathfrak{R}$ does. By induction, $\mathfrak{p}$ underlies $F_{k+1}$. Since $Q_{k} \in \mathfrak{R} \backslash \mathfrak{p}$, Lemma 7 shows that $\mathfrak{R}$ underlies $F_{k}$. This completes the proof of our proposition.

B. Proof of Theorem B. To prove our theorem, we may multiply by powers of the $x_{i}$ with $\left|\omega_{i}\right|=\|\omega\|$ to insure that the polynomials $R_{i}$ are of exact degree $D$. Set $g=\operatorname{dim} \mathfrak{A}, \Delta=\operatorname{deg} \mathfrak{A}$. Take a sequence $Q_{g}, \ldots, Q_{k+1}$ of maximal length $g-k$ such that each $Q_{\kappa}, g \geq \kappa \geq k+1$, satisfies the ensuing conditions (a) and (b) and such that the ideal $\mathfrak{B}_{k}$ has some isolated prime component $\mathfrak{D}$ of dimension $k$ with zeros in $B_{\rho}(\omega)$.

(a) $Q_{\kappa}=\sum a_{\kappa i} R_{i}, a_{\kappa i} \in \mathbf{Z}$ with $\left|a_{\kappa i}\right| \leq \Delta D^{g-\kappa}$, and

(b) $Q_{\kappa}$ does not lie in any isolated prime ideal of $\mathfrak{B}_{\kappa}:=\left(\mathfrak{A}, Q_{g}, \ldots, Q_{\kappa+1}\right)$ of dimension $\kappa$ having zeros in $B_{\rho}(\omega)$.

Since $\mathfrak{D}$ has a zero in $B_{\rho}(\omega), g-k \leq g$. Note that to satisfy (b), the polynomials $Q_{g}, \ldots, Q_{k+1} \in \mathfrak{D}$ are linearly independent combinations of the $R_{i}$. Since the $R_{i}$ have no common zero in $B_{\rho}(\omega), g-k<n$. Thus $g-k \leq \mu$.

Notice that according to Theorem II of [Ma-Wü] or Proposition 3.3 of [Ph4] (or [Br4]), the number of isolated prime ideals of $\mathfrak{B}_{k}$ of dimension $k$ is at most $\Delta D^{g-k}$. Since the $R_{i}$ have no zeros in common with $\mathfrak{A}$ inside $B_{\rho}(\omega)$, then according to, say, [Ma-Wü, Lemma 2, p. 438], there is a Z-linear combination

$$
Q_{k}=a_{k 1} R_{1}+\cdots+a_{k n} R_{n},
$$

$\left|a_{k i}\right| \leq \Delta D^{g-k}$, lying outside all the isolated prime components of $\mathfrak{B}_{k}$ which have zeros inside $B_{\rho}(\omega)$, and at most $\Delta D^{g-k}$ of the $a_{k i}$ are nonzero.

Thus by the maximality of the length of the sequence $Q_{g}, \ldots, Q_{k+1}$, we conclude that $\mathfrak{B}_{k-1}=\left(\mathfrak{A}, Q_{g}, \ldots, Q_{k}\right)$ has no isolated components of dimension $k-1$ with zeros in $B_{\rho}(\omega)$. We now apply the Proposition with $d=g+1$ and express the $Q_{\kappa}$ in terms of the $P_{i}$ to establish the theorem.

C. Proof of Theorem A and Corollary B. We consider the homogenizations (to fixed degree $D$ ) of our polynomials $P_{i}$ :

$$
{ }^{h} P_{i}\left(x_{0}, \ldots, x_{m}\right)=P_{i}\left(x_{1} / x_{0}, \ldots, x_{m} / x_{0}\right) x_{0}{ }^{D} .
$$


Then for $\omega^{*}=\left(1, \omega_{1}, \ldots, \omega_{m}\right)$,

$$
\left\|^{h} P\right\|_{\omega^{*}}=|P(\omega)| /\left\|\omega^{*}\right\|^{\operatorname{deg} P} \leq|P(\omega)|,
$$

where $\left\|\omega^{*}\right\|=\max \left\{1,\left|\omega_{i}\right|\right\}=|\omega|$. Let $\theta^{*}=\left(1, \theta_{1}, \ldots, \theta_{m}\right) \in B_{\varepsilon}\left(\omega^{*}\right), \varepsilon>0$. Then $\max \left\{1,\left|\theta_{i}\right|\right\}=:\left\|\theta^{*}\right\|<\varepsilon\left\|\omega^{*}\right\|\left\|\theta^{*}\right\|+\left\|\omega^{*}\right\|$, whence

$$
\max \left|\omega_{i}-\theta_{i}\right|<\varepsilon\left\|\omega^{*}\right\|^{2} /\left(1-\varepsilon\left\|\omega^{*}\right\|\right)<2 \varepsilon|\omega|^{2},
$$

if $\varepsilon<1 / 2\left\|\omega^{*}\right\|$. Moreover any $\theta_{*}=\left(0, \theta_{1}, \ldots, \theta_{m}\right) \notin B_{1 /\left\|\omega^{*}\right\|}\left(\omega^{*}\right)$. Thus since the $P_{i}$ have no common zero within $\rho$ of $\omega, 0<\rho \leq 1$, we conclude that if the ${ }^{h} P_{i}$ have a common zero in $B_{\varepsilon}\left(\omega^{*}\right)$, then $\varepsilon>\rho / 2 m^{1 / 2}|\omega|^{2}=: \rho^{\prime}$.

Now we apply Theorem B with $\rho^{\prime}$ for $\rho, \omega^{*}$ for $\omega$. For Theorem A we set $\mathfrak{A}=(0)$, and for Corollary $B$, we take $\mathfrak{A}$ to be the homogenization ${ }^{h} \mathfrak{B}$. In both cases $\|\mathfrak{A}\|_{\omega^{*}}=0$.

D. Proof of Theorem $\mathrm{A}^{\prime}$. This proof is quite analogous to that of Theorem A with two differences. First of all we work in $\mathbf{Z}\left[y, x_{1}, \ldots, x_{m}\right]$ instead of $\mathbf{Z}\left[x_{1}, \ldots, x_{m}\right]$, and $\left(\zeta, \omega_{1}, \ldots, \omega_{m}\right)$ is our point. Secondly $\mathfrak{A}=$ $\left(x_{0}{ }^{\operatorname{deg} f} f\left(y / x_{0}\right)\right)$, where $f$ is the minimal polynomial for $\zeta$ over $\mathbf{Z}$. Then the proof proceeds as before with the remark that the nearest conjugate to $\zeta$ lies at least $\rho$ away from $\zeta$. Therefore suitable $c_{1}{ }^{\prime \prime}, c_{2}{ }^{\prime \prime}$ can be read off from Corollary B.

\section{BIBLIOGRAPHY}

[Br1] W. D. Brownawell, Some remarks on semi-resultants, Transcendence Theory: Advances and Applications, Chapter 14 (A. Baker and D. W. Masser, eds.), New York, Academic Press, 1977.

[Br2] __ Bounds for the degree in the Nullstellensatz, Annals of Math. 126 (1987), 577-591.

[Br3] __ Large transcendence degree revisited I. Exponential and non-CM cases, Bonn Transcendence Workshop 1985 (G. Wüstholz, ed.), Lecture Notes in Math., Springer-Verlag, Berlin, New York and Heidelberg (to appear).

[Br4] _ Note on a paper of P. Philippon, Michigan Math. J. 34 (1987) (in press).

[Br-Tu] W. D. Brownawell and R. Tubbs, Large transcendence degree revisited II. The CM case, Bonn Transcendence Workshop 1985 (G. Wüstholz, ed.), Lecture Notes in Math., SpringerVerlag, Berlin, New York and Heidelberg (to appear).

[Ch] G. V. Chudnovsky, Some analytic methods in the theory of transcendental numbers, Inst. of Math., Ukr. SSR Acad. Preprints IM 74-8 and IM 74-9, Kiev, $1974=$ Chapter 1 in Contributions to the Theory of Transcendental Numbers, Math. Surveys Monogr., Vol. 19, Amer. Math. Soc., Providence, R. I., 1984.

[Ge] A. O. Gelfond, Transcendental and algebraic numbers, New York, Dover, 1960.

[Ma-Wü] D. W. Masser and G. Wüstholz, Fields of large transcendence degree generated by values of elliptic functions, Invent. Math. 72 (1971), 407-463.

[Nel] Yu. V. Nesterenko, Estimates for the orders of zeros of functions of a certain class and their applications in the theory of transcendental numbers, Izv. Akad. Nauk SSSR Ser. Mat. 41 (1977), 253-284 = Math. USSR Izv. 11 (1977), 239-270.

[Ne2] _ Bounds for the characteristic function of a prime ideal, Mat. Sbornik 123(165), No. 1 (1984), 11-34 = Math. USSR Sbornik 51 (1985), 9-32. 
[Ne3] _ _ On the algebraic independence of algebraic powers of numbers, Mat. Sbornik 123(165), No. 4 (1984), 435-459 = Math. USSR Sbornik 51 (1985), 429-454, brief version in Approximations Diophantiennes et Nombres Transcendants, Boston-Basel-New York, Birkhäuser Verlag, 1983, pp. 199-220.

[Ne4] _ , On the measure of algebraic independence of the values of several functions, Mat. Sbornik 128(170), No. 4 (1985), 545-568 = Math. USSR Sbornik 56 (1986), 545-567.

[Ph1] P. Philippon, Critères pour l'indépendance algébrique, Inst. Hautes Études Sci. Publ. Math. 64 (1986), 5-52.

[Ph2] __ Sur les mesures d'indépendance algébrique, Séminaire de Théorie des Nombres, BostonBasel-Stuttgart, Birkhäuser, 1985, pp. 219-233.

[Ph3] _ Elimination effective, Journées Algorithmiques-Arithmétiques, Chap. XXVIII, Publ. Univ. St. Etienne, France, 1983.

[Ph4] _ Lemmes de zéros dans les groupes algébriques commutatifs, Bull. Soc. Math. France 114 (1986), 355-383.

Department of Mathematics, Pennsylvania State University, University Park, PennSYLVANIA 16802 